\title{
NUEVAS OBSERVACIONES DE RODOFÍCEAS EN AGUAS EPICONTINENTALES DEL NE Y SE DE ESPAÑA
}

\author{
S. Sabater ${ }^{1}$, M. Aboal $^{2}$ y J. Cambra ${ }^{3}$ \\ 1. Departament d'Ecologia, Facultat de Biologia, Universitat de Barcelona. Avda. Diagonal, 645, 08028 Barce- \\ lona. Spain. \\ 2. Departamento de Biología Vegetal, Unidad de Botánica, Facultad de Biología, Universidad de Murcia. Cam- \\ pus de Espinardo, 30071 Murcia. Spain. \\ 3. Departament de Biologia Vegetal, Unitat de Botanica Sistemàtica i Geobotanica, Facultat de Biologia, Uni- \\ versitat de Barcelona. Avda. Diagonal, 645, 08028 Barcelona. Spain.
}

Palabras clave: Rhodophyceae, distribution, autoecology, Spain

\author{
ABSTRACT \\ NEW RHODOPHYCEAE RECORDS FOR THE NE AND SE SPANISH CONTINENTAL \\ WATERS
}

New localities, taxonomical comments and ecological data are given for twelve species of Rhodophyta, that have been collected in NE and SE Spain during phycological surveys. Among them we can point out some specially interesting observations concerning to Bangia atropurpurea, Thorea ramosissima, Compsopogon coeruleus and Lemanea nodosa.

\section{INTRODUCCIÓN}

Los rodófitos de aguas dulces han sido poco estudiados, principalmente a causa de la dificultad de identificación de las especies y a la ausencia de monografías adecuadas sobre el tema. En el área que comprende el presente estudio, la vertiente mediterránea de la Península Ibérica, se han citado 18 especies hasta el momento (CAMBRA, 1985, 1987), número relativamente bajo si lo comparamos con las treinta y cinco especies encontradas en la vertiente atlántica portuguesa (REIS, 1974) y las cuarenta y ocho que se cono-

Limnética, 5: 93-100 (1989)

(C) Asociación Española de Limnología, Madrid, Spain cen en Francia (HAMEL, 1924, 1925; BourRelly, 1970). En realidad, solamente unas ciento ochenta especies de veintinueve géneros distintos se distribuyen en las aguas dulces (SHEATH, 1984). Ello constituye un 3,4\% del total aproximado de rodofíceas conocidas.

A pesar de este número aparentemente pobre de especies, las rodofíceas son importantes por su amplia distribución, principalmente en las aguas corrientes. Así, en latitudes templadas, se han hallado en un 50-95\% de los ríos (SheAth, 1984). En este trabajo aparecen nuevos datos de la distribución, autoecología y taxonomía de las especies observadas en el área mediterránea de la Península Ibérica. 


\section{MATERIAL Y MÉTODOS}

Durante estudios algológicos extensivos realizados en el SE de España (АвOAL, 1987) así como en la cuenca fluvial del río Ter, en el NE (SABATER, 1987), se han realizado observaciones de distintas especies de rodofíceas, que se han venido a añadir a otras más ocasionales en el área que cubre la franja E de la Península Ibérica. Así, se han estudiado más de sesenta localidades, que cubren un amplio rango de hábitats, como arroyos, ríos, fuentes y otros ambientes subaéreos, en una gran variedad de substratos y de condiciones fisico-químicas. En todos los casos, las muestras se fijaron in situ con solución de formol al $4 \%$. En la mayor parte de los casos, la temperatura, el pH y la conductividad se midieron en el campo, y se recogieron muestras de agua para la determinación de la alcalinidad y de la concentración de nutrientes (fosfatos y nitratos) (tabla 1). Los métodos utilizados están referidos en APHA (1980).

\section{RESULTADOS}

\section{Chroothece rupestris Hansgirg}

Se desarrolla en forma de colonia arbuscular, poco definida, al principio verdosa y más tarde parduzca, generalmente pedunculada. Las células son de 7-10 $\mu \mathrm{m}$ de ancho por 9-20 $\mu \mathrm{m}$ de largo, cada una rodeada por una cubierta gelatinosa, es- tratificada, hialina y gruesa (hasta $2 \mu \mathrm{m}$ de ancho), con un cromatóforo axial y de forma estrellada poco definida (fig. la). Puede confundirse fácilmente con Pseudoncobyrsa rivularis Geitler, una cianofícea que vive en los mismos ambientes y que tiene un tipo morfológico convergente con ella. Como observa StaRmach (1977), acostumbra a desarrollarse sobre rocas húmedas, principalmente en aguas alcalinas y poco iluminadas. MARGALEF (1955) la señala en pequeños torrentes y fuentes de la montaña media de Cataluña. La hemos observado en unas pocas localidades, siempre epilítica en cursos de agua alcalinos y de corriente débil (tabla 2).

Chroodactylon ramosum (Thwaites) Hansgirg

Las células se alinean en una vaina gelatinosa y amplia, formando un filamento uniseriado, irregularmente ramificado; son elipsoidales, de 12$25 \mu \mathrm{m}$ de longitud por $10 \mu \mathrm{m}$ de anchura, con un plasto axial verdeazulado y estrellado, provisto de un grueso pirenoide (fig. lb).

Esta especie fue previamente señalada en nuestro país por GonZÁlez Guerrero (1946) como Asterocytis ramosa, por LewIN (1888) como A. wolleana y por MARGALEF (1951, 1955) como A. ornata y $\boldsymbol{A}$. ramosa. Ha sido observada como epífita sobre algas filamentosas, especialmente Cladophora, muy extendida en aguas corrientes y fuentes de aguas dulces y salobres del SE (tabla 2). La tolerancia de esta especie frente a la salinidad ya fue señalada por STARMACH (1977).

Tabla 1.- Valores extremos observados de los principales parámetros fisico-químicos de las aguas en que fueron halladas las especies de rodofíceas que se relacionan.

Extreme values for the main physico-chemical parameters of waters where the mentioned Rhodophyceae species were found.

\begin{tabular}{|c|c|c|c|c|c|c|c|}
\hline Especies & $p H$ & $\begin{array}{c}\text { Temperatura } \\
\left({ }^{\circ} \mathrm{C}\right)\end{array}$ & $\begin{array}{c}\text { Conductividad } \\
(\mu \mathrm{S} / \mathrm{cm})\end{array}$ & $\begin{array}{c}\text { Alcalinidad } \\
\text { (meq } / l)\end{array}$ & $\begin{array}{c}\text { Cloruros } \\
(\mathrm{mg} / \mathrm{l})\end{array}$ & $\begin{array}{c}\text { Nitratos } \\
(\mu M)\end{array}$ & $\begin{array}{c}\text { Fosfatos } \\
(\mu M)\end{array}$ \\
\hline Audouinella pygmaea & $6,5-8,2$ & $12-28$ & $211-1.200$ & $3,4-7,1$ & $19-357$ & $0-124$ & $0,00-31,6$ \\
\hline Audouinella violacea & $6,5-8,1$ & - & $292-2.100$ & $3,8-7,1$ & $19-263$ & $0-123$ & $0-12$ \\
\hline Bangia atropurpurea & 8,0 & $9-10$ & $198-288$ & $2,4-2,5$ & - & $27-87$ & $2,6-3,4$ \\
\hline Batrachospermum moniliforme & $6,5-8,2$ & - & $250-500$ & $3,4-7,1$ & $22,6-188$ & $0-123$ & $0-0,9$ \\
\hline Chroothece rupestris & $6,5-8,2$ & - & $250-1.110$ & $4-6$ & $24-150$ & $2,4-124$ & $0-0,4$ \\
\hline Chroodactylon ramosum & $6,5-8,4$ & $16-28$ & $296-25.000$ & $3-17$ & $24-21.800$ & $2,4-176$ & $0-12$ \\
\hline Compsopogon coeruleus & $7,6-8,2$ & - & $250-1.110$ & $4-6$ & $24-150$ & $2,4-124$ & $0-0,4$ \\
\hline Hildenbrandia rivularis & $6,4-9,2$ & $13-15$ & $150-3.056$ & $0,2-1,6$ & - & - & - \\
\hline Lemanea fluviatilis & $6,4-7,9$ & $6-12$ & $392-2.250$ & $0,6-1,3$ & & & \\
\hline Lemanea nodosa & $7,7-8,2$ & $14,14,5$ & 104 & - & - & - & - \\
\hline Thorea ramosissima & 8,00 & 24,5 & 996 & 2,9 & - & 99,7 & 2,72 \\
\hline
\end{tabular}


Bangia atropurpurea (Roth) C.A. Agardh

Filamento no ramificado, de 54-60 ym de ancho, biseriado en la parte basal y multiseriado en la apical (fig. lc). Las células, rodeadas por una cubierta hialina y gruesa, son de 7-10 $\mu \mathrm{m}$ de ancho por 41-43 $\mu \mathrm{m}$ de largo, rectangulares en la base de la plantita y cuadradas-redondeadas en el ápice, donde su diámetro es de 18-23 ym.

Ha sido observada en el río Ter, en un lugar de aguas rápidas. En el momento en que fue hallada, las aguas eran particularmente frías y ricas en nutrientes (tabla 1). Se trata de una rodofícea común en los tramos altos de los ríos de Europa (Scannell, 1972; Descy, 1973; Friedrich \& MuLLER, 1984), pero que no lo parece tanto en los nuestros. De hecho, aunque se ha observado en algunos ríos caudalosos del Pirineo catalán duran- te la primavera (MARGalef, com. pers.) sólo había sido citada en España previamente en tres ocasiones: GonZález Fragoso (1883) en Sevilla, LACOIZQueta (1885) en Navarra y CABAllero (1929) en Guadalajara. Antiguamente, se hablaba de dos especies en este género, una marina, y otra de agua dulce. Recientemente, se ha comprobado que son, de hecho, una única especie (Sheath \& Cole, 1984), que crece en los dos ambientes. Se trata de una especie oportunista, propia de los estuarios y del mar, pero que puede invadir secundariamente las aguas dulces (SHEATH, 1984).

\section{Compsopogon coeruleus (Balbis) Montagne}

Ha sido observada en el NE de España por BusQUETs et al. (1985) y TOMAs et al. (1980), distri-
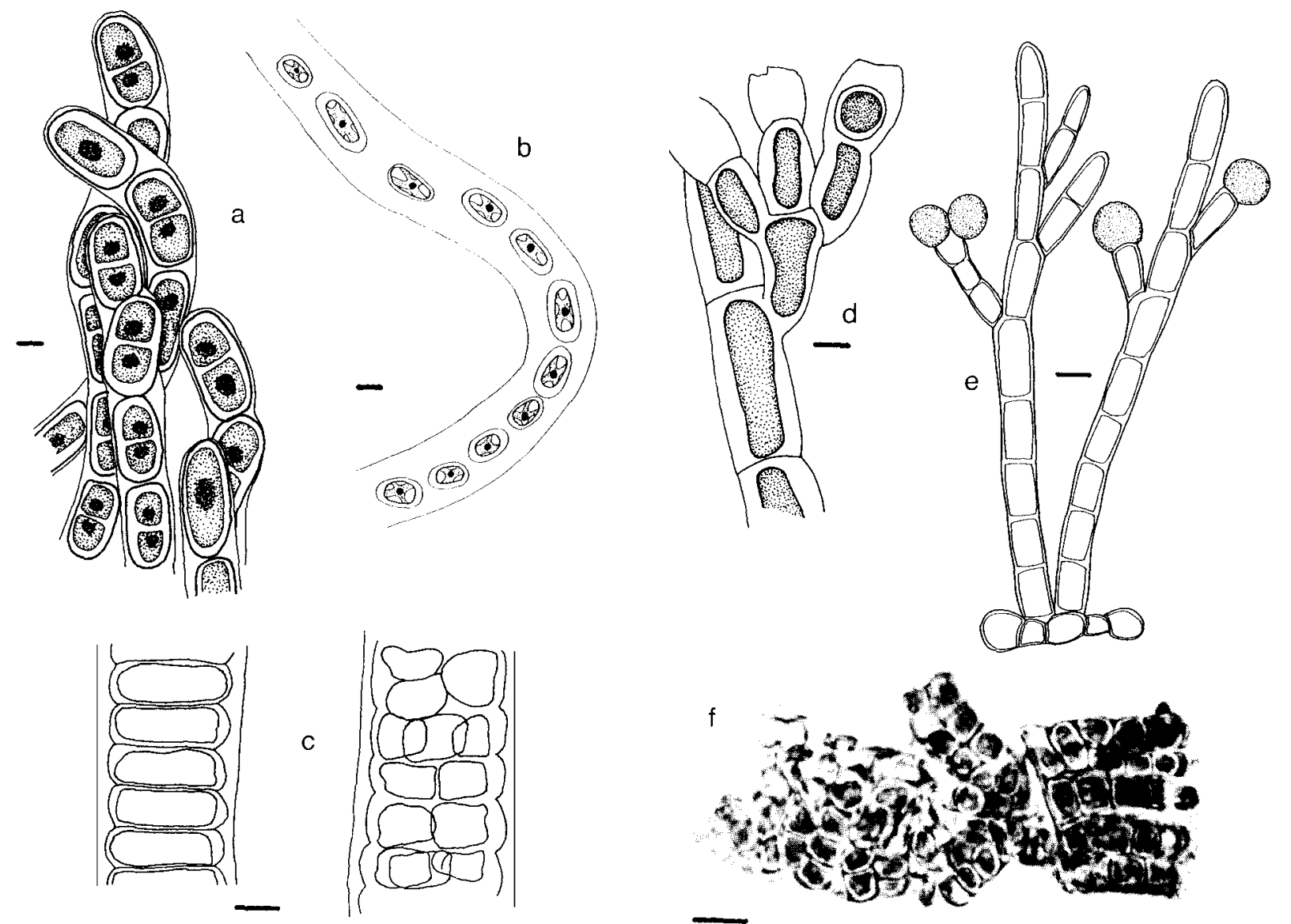

Figura 1.- a) Choothece rupestris; b) Chroodactylon ramosum;c) Bangia atropurpurea; d) Audouinella leiblenii; e) Audouinella pygmaea; f) Hildenbrandia rivularis (barra $=10 \mu \mathrm{m}$ ). 
Tabla 2.- Localidades del E de la Península Ibérica en que se han observado especies de rodofíceas epicontinentales. En cada caso se indica la provincia, el lugar, las coordenadas UTM y el hábitat.

Localities of Eastern Iberian Peninsula where epicontinental Rhodophyceae species were found. Each case shows province, place, UTM references and habitat.

\begin{tabular}{|c|c|c|c|c|}
\hline Localidad & U.T.M. & Habitat & Fecha & Especies \\
\hline 1. ALBACETE: río Madera & 30 SWH3332 & Epiliton & $\mathrm{VI} / 83$ & $\begin{array}{l}\text { Chroothece rupestris } \\
\text { Batrachospermum moniliforme } \\
\text { Audouinella pygmaea } \\
\text { Audouinella violacea }\end{array}$ \\
\hline 2. ALBACETE: arroyo de Tus & 30SWH4951 & Epiliton & VIII/85 & Batrachospermum moniliforme \\
\hline 3. ALBACETE: arroyo de la Vega & 30 SWH5160 & Epiliton & $\mathrm{VII} / 83$ & Audouinella pygmaea \\
\hline 4. ALBACETE: río de las Hoyas & 30 SWH5670 & Epiliton & $\mathrm{VII} / 85$ & $\begin{array}{l}\text { Audouinella pygmaea } \\
\text { Audouinella violacea }\end{array}$ \\
\hline 5. ALBACETE: río de las Hoyas & 30 SWH5369 & Epiliton y epífito de Cladophora & $\mathrm{VII} / 83$ & $\begin{array}{l}\text { Chroothece rupestris } \\
\text { Chroodactylon ramosum } \\
\text { Batrachospermum moniliforme }\end{array}$ \\
\hline 6. ALBACETE: río Mundo & 30 SWH6262 & Epiliton & $\mathrm{VII} / 83$ & Audouinella pygmaea \\
\hline 7. ALBACETE: río Tus & 30 SWH5951 & Epiliton y epífito de Cladophora & $\mathrm{VII} / 83$ & $\begin{array}{l}\text { Chroothece rupestris } \\
\text { Chroodactylon ramosum } \\
\text { Batrachospermum moniliforme }\end{array}$ \\
\hline 8. ALBACETE: río Taibilla & 30SWH6331 & Epiliton & $\mathrm{VII} / 83$ & $\begin{array}{l}\text { Audouinella violacea, } \\
\text { Audouinella pygmaea }\end{array}$ \\
\hline 9. ALBACETE: río Endrinales & 30 SWH5467 & Epiliton & $\mathrm{VII} / 85$ & Audouinella pygmaea \\
\hline 10. ALBACETE: no Mundo & 30 SWH7767 & Epiliton & VIII/85 & $\begin{array}{l}\text { Chroodactylon ramosurn } \\
\text { Audouinella violacea }\end{array}$ \\
\hline 11. ALBACETE: río Mundo & 30 SWH8067 & Epiliton & $\mathrm{VII} / 83$ & Audouinella pygmaea \\
\hline 12. ALBACETE: fuente del Hueso & $30 \mathrm{SXH} 2366$ & Epífito de Cladophora & $\mathrm{VII} / 83$ & Chroodactylon ramosum \\
\hline 13. ALBACETE: arroyo Fuentes & 30 SWH 5673 & Epiliton & $\mathrm{VII} / 83$ & Batrachospermum moniliforme \\
\hline 14. ALBACETE: río Acequias & 30 SWH5673 & Epiliton & $\mathrm{VII} / 83$ & Audouinella pygmaea \\
\hline 15. ALBACETE: río de las Hoyas & 30 SWH 7767 & Epiliton & $\mathrm{VII} / 83$ & Audouinella violacea \\
\hline 16. ALBACETE: no Madera & 30 SWH3162 & Epiliton & $\mathrm{VI} / 83$ & $\begin{array}{l}\text { Audouinella pygmaea } \\
\text { Audouinella violacea }\end{array}$ \\
\hline 17. ALBACETE: río Mencal & 30SWH6375 & Epiliton & $\mathrm{VII} / 83$ & Audouinella pygmaea \\
\hline 18. ALBACETE: río Viñazos & 30 SWH5974 & Epiliton & $\mathrm{VII} / 85$ & Audouinella pygmaea \\
\hline 19. ALBACETE: no Salado & 30 SWH 5260 & Epiliton y epífito de Cladophora & $\mathrm{VII} / 83$ & $\begin{array}{l}\text { Audouinella pygmaea, } \\
\text { Audouinella violacea, } \\
\text { Chroodactylon ramosum }\end{array}$ \\
\hline 20. ALBACETE: arroyo de Sierra & $30 \mathrm{SWH} 4849$ & Epiliton & $\mathrm{VII} / 83$ & $\begin{array}{l}\text { Audouinella pygmaea, } \\
\text { Audouinella violacea }\end{array}$ \\
\hline 21. ALACANT: río Segura & 30SYH0418 & $\begin{array}{l}\text { Epífito de los tallos de Arundo donax } \\
\text { y Phragmites communis; agua salobre }\end{array}$ & VIII/83 & Compsopogon coeruleus \\
\hline 22. ALACANT: río Segura & $30 \mathrm{SXH} 9717$ & $\begin{array}{l}\text { Epífito de los tallos de Arundo donax } \\
\text { y Phragmites communis; agua salobre }\end{array}$ & VIII/83 & Compsopogon coeruleus \\
\hline 23. ALACANT: laguna del Hondo & $30 \mathrm{~S} \times \mathrm{H} 9528$ & Pleuston & VIII/83 & Compsopogon coeruleus \\
\hline 24. BARCELONA: riera de Vallosera & 31 TDG3729 & Epiliton; en lugares sombríos & V/86 & Batrachospermum moniliforme \\
\hline $\begin{array}{l}\text { 25. BARCELONA: arroyos de las Guille- } \\
\text { ries }\end{array}$ & 31 TDG522458 & Epiliton; aguas rápidas y limpias & IV/84 & Hildenbrandia rivularis \\
\hline 26. BARCELONA: macizo de Cadiretes & $31 T D G 92$ & Epiliton; en lugares iluminados & $V / 86$ & Batrachospermum moniliforme \\
\hline 27. BARCELONA: riera de Vallvidrera & 31TDG08 & Epiliton & II $/ 85$ & Hildenbrandia rivularis \\
\hline 28. BARCELONA: riu Major & 31 TDG514430 & Epiliton & $\mathrm{XII} / 86$ & Hildenbrandia rivularis \\
\hline 29. GIRONA: fuente de l'Adou & 31 TEG18 & Epiliton & $\mathrm{V} / 86$ & Hildenbrandia rivularis \\
\hline 30. GIRONA: riera de l'Anyet & 31TDH9501 & Epiliton & IV $/ 86$ & Hildenbrandia rivularis \\
\hline 31. GIRONA: La Vola & 31 TDG428587 & Epiliton & III/86 & $\begin{array}{l}\text { Batrachospermum moniliforme, } \\
\text { Chroothece rupestris }\end{array}$ \\
\hline 32. GIRONA: río Ter (Bescanó) & $31 T D G 779468$ & Epiliton & $\mathrm{I} / 84$ & Bangia artropurpurea \\
\hline 33. GIRONA: río Ter (Torelló) & 31 TDG384546 & $\begin{array}{l}\text { Epiliton; aguas frías y ricas en nu } \\
\text { trientes }\end{array}$ & $\mathrm{X} / 83$ & Bangia artropurpurea \\
\hline 34. GIRONA: riera de les Lloses & 31 TDG328701 & Epiliton; aguas con circulación lenta & & Audouinella leiblenii \\
\hline
\end{tabular}




\begin{tabular}{|c|c|c|c|c|}
\hline Localidad & U.T.M. & Habitat & Fecha & Especies \\
\hline 35. GIRONA: riera de Merdas & $31 T D G 263775$ & Epiliton; aguas alcalinas & $\mathrm{XI} / 85$ & Audouineiia violacea \\
\hline 36. GIRONA: río Gurri & 31TDG3154 & Epiliton; aguas alcalinas & $\mathrm{XI} / 85$ & Audouinelia violacea \\
\hline 37. GIRONA: arroyos de Cap de Creus & 31 TEG18 & $\begin{array}{l}\text { Epiliton; aguas corrientes frías y poco } \\
\text { mineralizadas }\end{array}$ & $\mathrm{VII} / 82$ & $\begin{array}{l}\text { Lemanea fluviatilis, } \\
\text { Hildenbrandia rivularis }\end{array}$ \\
\hline 38. GIRONA: riera de Viladrau & 31 TDG4833 & $\begin{array}{l}\text { Epiliton; aguas frías y poco minerali- } \\
\text { zadas }\end{array}$ & $\mathrm{V} / 85$ & Lemanea fluviatilis \\
\hline 39. GIRONA: arroyos de l'Albera & $31 \mathrm{TDH} 9501$ & Epiliton; aguas rápidas & $\mathrm{VI} / 84$ & Lemanea nodosa \\
\hline 40. JAEN: arroyo Romagillos & 30 SWH3230 & Epiliton; epífito de Cladophora & $\mathrm{VII} / 83$ & $\begin{array}{l}\text { Chroothece rupestris, } \\
\text { Batrachospermum moniliforme, } \\
\text { Chroodactylon ramosum }\end{array}$ \\
\hline 41. JAEN: río Madera & 30 SWH 3637 & Epiliton & VIII/85 & Batrachospermum moniliforme \\
\hline 42. JAEN: río Madera & 30 SWH3332 & Epiliton & $\mathrm{VI} / 83$ & $\begin{array}{l}\text { Chroothece rupestris, } \\
\text { Batrachospermum moniliforme, } \\
\text { Audouineila pygmaea, } \\
\text { Audouinella violacea }\end{array}$ \\
\hline 43. JAEN: embalse de Anchuricas & 30 SWH 4128 & Epiliton & VIII/85 & Batrachospermum moniliforme \\
\hline 44. JAEN: río Madera & 30 SWH3436 & Epiliton & $\mathrm{VI} / 83$ & $\begin{array}{l}\text { Batrachospermum moniliforme, } \\
\text { Audouinella pygmaea, } \\
\text { Audouinella violacea }\end{array}$ \\
\hline 45. JAEN: arroyo de Rocanales & 30 SWH3333 & Epiliton & VIII/85 & Batrachospermum moniliforme \\
\hline 46. LLEIDA: arroyos de Gerri de la Sal & $31 \mathrm{TCG} 4188$ & $\begin{array}{l}\text { Epiliton; aguas corrientes frías y poco } \\
\text { mineralizadas }\end{array}$ & $\mathrm{VII} / 82$ & Lemanea fluviatilis \\
\hline 47. MURCIA: río Segura & $30 \mathrm{~S} \times \mathrm{H} 4821$ & Epiliton & VIII/83 & Audouineila pygmaea \\
\hline 48. MURCIA: fuente Aceniche & $30 \mathrm{~S} \times \mathrm{H} 2204$ & Epífito de Cladophora & $\mathrm{V} / 83$ & Chroodactylon ramosum \\
\hline 49. MURCIA: fuentes del Marqués & 30SWH9817 & Epiliton & $\mathrm{VI} / 82$ & Batrachospermum moniliforme \\
\hline 50. MURCIA: río Benamor & $30 \mathrm{~S} \times \mathrm{H} 0030$ & Epífito de Cladophora & III/84 & Chroodactylon ramosum \\
\hline 51. MURCIA: río Benamor & 30 SWH 8829 & Epiliton y epífito de Cladophora & $\mathrm{XI} / 85$ & $\begin{array}{l}\text { Chroodactylon ramosum, } \\
\text { Chroothece rupestris }\end{array}$ \\
\hline 52. MURCIA: río Benamor & 30 SXH9130 & Epiliton y epífito de Cladophora & $\begin{array}{l}\text { IV/83 } \\
\text { IV/84 }\end{array}$ & $\begin{array}{l}\text { Chroodactylon ramosum } \\
\text { Chroothece rupestris, } \\
\text { Batrachospermum moniiiforme, } \\
\text { Audouinelia pygmaea }\end{array}$ \\
\hline 53. MURCIA: rambla Pcrea & $30 \mathrm{SXH} 3116$ & Epífito de Cladophora & III $/ 83$ & Chroodactylon ramosum \\
\hline 54. MURCIA: rambla del Tinajón & $30 \mathrm{~S} \times \mathrm{H} 5021$ & $\begin{array}{l}\text { Epífito sobre Cladophora, en agua sa- } \\
\text { lobre }\end{array}$ & IV $/ 84$ & Chroodactyion ramosum \\
\hline 55. MURCIA: río Segura & $30 \mathrm{SXH} 5267$ & Epiliton & $\mathrm{IX} / 83$ & Audouinella violacea \\
\hline 56. MURCIA: río Mula & $30 \mathrm{SXH1709}$ & Epífito de Cladophora & III $/ 83$ & Chroodactylon ramosum \\
\hline 57. MURCIA: río Benamor & 30 SWH 9030 & Epiliton & $\mathrm{VII} / 83$ & Audouinella pygmaea \\
\hline 58. MURCIA: rambla del Carrizalejo & $30 S \times H 5326$ & Epiliton, en aguas salobres & $\mathrm{VII} / 83$ & Compsopogon coeruleus \\
\hline 59. MURCIA: rambla del Saladar & $30 \mathrm{SXH} 5125$ & $\begin{array}{l}\text { Epífito de Cladophora, en aguas salo- } \\
\text { bres }\end{array}$ & IV/84 & Chroodactyion ramosum \\
\hline 60. TARRAGONA: río Ebro & 31TBF9132 & $\begin{array}{l}\text { Aguas de conductividad elevada y li- } \\
\text { geramente turbias }\end{array}$ & $\mathrm{VII} / 87$ & Thorea ramosissima \\
\hline
\end{tabular}

buida por aguas dulces y salinas. En el presente trabajo se ha hallado en numerosas localidades (tabla 2) de aguas salinas interiores o con influencia marina del SE de España, conviviendo con Enteromorpha sp. pl. (Chlorophyceae), y tolerando una carga orgánica relativamente importante (tabla 1). Ha sido principalmente observada como epífita sobre raicillas de Phragmites australis y Arundo donax, aunque también como epilítica.
Audouinella leiblenii (Kützing) Sheath

Células de 15-(17) $\mu \mathrm{m}$ de diámetro y 50-70 $\mu \mathrm{m}$ de longitud. Monósporas de $12 \mu \mathrm{m}$ de diámetro (fig. 1d).

Fue observada en un pequeño río calcáreo de circulación lenta (tabla 2), con abundantes detritus vegetales y muy sombrío. Los talos crecían donde la corriente era poco intensa, constituyendo masas esféricas de 2-4 mm de diámetro, sobre 
los cantos rodados o bien epífitos sobre Cladophora glomerata. Los filamentos estaban cubiertos por gran cantidad de incrustaciones minerales y de valvas de la diatomea Cocconeis pediculus. Esta especie, cosmopolita según STARMACH (1977), sólo había sido observada por MARGALEF (1955) en un pequeño canal de Riells del Fai (Barcelona). Como otras especies del mismo género, prefiere las aguas ricas en carbonato cálcico, de corriente suave y que reciben poca luz.

\section{Audouinella pygmaea (Kützing) Aboal}

Células vegetativas de 13-25 $\mu \mathrm{m}$ de longitud y $10 \mu \mathrm{m}$ de anchura. Monósporas de 9-11 por 10$13 \mu \mathrm{m}$ (fig. 1, e).

Esta especie, de distribución mundial (STARMACH, 1977), es relativamente abundante en todo el SE español (tabla 2), como componente del pecton, en pequeños saltos de agua, condiciones de alcalinidad elevada y moderadas concentraciones de cloruros (tabla 1).

\section{Audouinella violacea (Kützing) Hamel}

Se trata de una especie muy común, que habita en cursos de agua de elevada reserva alcalina (tabla 1). Ha sido hallada con frecuencia, tanto en el NE como en el SE (tabla 2).

\section{Batrachospermum moniliforme Roth}

Especie muy ampliamente distribuida, que crece óptimamente tanto en circunstancias de alta como de baja iluminación (RIDER \& W AGNER, 1972; Sheath \& Cole, 1984). Ha sido observada principalmente en las aguas corrientes y limpias de montañas de baja y media altitud, casi siempre sobre sustrato calcáreo (tabla 1). En Cataluña ha sido encontrada en diversos torrentes de pequeños macizos, así como en fuentes. En el SE, es muy frecuente sobre rocas, o epífita sobre macrófitos, formando poblaciones importantes en algunos puntos (tabla 2).

\section{Lemanea fluviatilis (Dillw.) Ag.}

Es la especie más común del género, propia de aguas corrientes, frías y poco mineralizadas (tabla 1). Es frecuente observarla en masas importantes tapizando las rocas de los rápidos, generalmente en zonas poco iluminadas. Ha sido encontrada en diversos cursos de agua del NE español (tabla 2).

\section{Lemanea nodosa Kütz.}

Esta especie ha sido observada en un torrente de aguas poco mineralizadas (tabla 1 ) de la provincia de Gerona (tabla 2). Los talos eran muy abundantes en la zona de corriente. Anteriormente, era tan sólo conocida del Torrent d'Arbúcies (Barcelona) (CAMBRA, 1987).

\section{Thorea ramosissima Bory}

Observada, creciendo en abundancia, sobre piedras sumergidas en una orilla forestada del río Ebro, en aguas poco rápidas y de conductividad elevada (tabla 1). La aparición de esta especie fue muy puntual, y estuvo circunscrita a un momento de turbidez muy escasa del agua. Su distribución parece limitarse a una pequeña área mediterránea, pues tan sólo se había hallado previamente en Pego, Valencia (Tomas, 1981).

\section{Hildenbrandia rivularis (Liebmann) Agardh}

Esta especie incrustante (fig. lf) ha sido observada en aguas rápidas y limpias del NE. Se encuentra muy ampliamente difundida en los ríos y fuentes de montaña, formando manchas rojizas sobre las rocas sumergidas (tabla 2).

Parece ser indiferente al tipo de substrato en el que vive (calcáreo o silíceo), y sus preferencias se centran en la baja iluminación (BourRelLy, 1955). De hecho, la variedad de condiciones ambientales en que la hemos observado es bastante amplia (tabla 1), pero siempre en áreas forestadas.

La comunidad formada por este rodófito es, en nuestro país, monoespecífica. MARGalef (1950) la nombró como Hildenbrandietum rivularis. A diferencia de lo que describe Fritsch (1929) en los ríos ingleses, o KANN (1978) en los austríacos, no aparece nunca en compañía del feófito Lithoderma fluviatile Aresch. [= Heribaudiella fluviatilis (Aresch.) Sved.]. Estas dos especies aparecen conjuntamente también en pequeños ríos de Baviera (Alemania Federal) (SABATER, datos no publicados). Curiosamente, el feófito aparece en los mismos hábitats en el E de América del Norte, pero sin Hildenbrandia rivularis (WeHR \& SteIN, 1985). Aunque es posible que en algún caso la existencia de una u otra especie se haya pasado por alto, su distribución parece un interesante problema biogeográfico. En el E de España, los acompañantes de la rodofícea son, básica- 
mente, cianofíceas incrustantes (SABATER, 1987), análogamente a lo que observa STARMACH (1963, 1969), o bien líquenes del género Verrucaria, como ha observado CAMBra (1986) en el cabo de Creus.

\section{DISCUSIÓN}

Las rodofíceas no marinas se distribuyen principalmente en aguas lóticas, oxigenadas y relativamente frías, aunque algunas pueden encontrarse en aguas más tranquilas. El conjunto de estos rodófitos continentales, en comparación con los marinos, se caracteriza por la simplificación, no tan sólo de su estructura, sino también de sus ciclos vitales. Ello ha llevado a pensar que las especies de agua dulce podrían provenir de otras marinas que hubiesen invadido los ambientes lóticos en tiempos recientes, o bien que su origen no fuere monofilético, sino que una parte de ellas proviniese de la evolución de algunas formas de cianofíceas de los ríos (SKUjA, 1983). Todas estas consideraciones están, sin duda, en la base de los principales problemas en la distribución y la ecología de estas algas.

Algunas de las especies observadas en el E de la Península Ibérica son cosmopolitas (Batrachospermum moniliforme), pero la mayor parte tienen preferencias específicas que justifican su distribución más limitada. Thorea ramosissima y Compsopogon coeruleus parecen tener su óptimo en aguas tropicales (STARMACH, 1977), y por esta razón su aparición en latitudes templadas está limitada a canales o lagunas, lugares donde el agua puede alcanzar en verano altas temperaturas (BAtтiAto et al., 1979). Esta preferencia podría asimismo relacionarse con la mayor eficiencia fotosintética que presentan estas especies a $30-35^{\circ} \mathrm{C}$ de temperatura, mientras que otras distribuidas en latitudes templadas, como Lemanea fluviatilis, lo poseen a $15{ }^{\circ} \mathrm{C}$ (KREMER, 1983). Esta última especie prefiere, además, aguas corrientes de velocidad elevada. Ambas exigencias se pueden encontrar en las aguas frías de montaña, que en nuestra área de estudio están limitadas a pequeñas áreas del NE.

Mientras que algunas especies pueden habitar en aguas de distinta conductividad y pH (Audoui- nella sp. pl.: tabla 1), otras como Bangia atropurpurea son más exigentes. SHEAth (1984) señala que en Estados Unidos esta especie ha sido únicamente observada en aguas con conductividades entre 200 y $550 \mu \mathrm{S} / \mathrm{cm}$.

La luz es uno de los factores que más afectan la distribución de las rodofíceas de agua dulce (Whitton, 1975). Mientras que algunas especies son eurifóticas (Batrachospermummoniliforrne), otras precisan un estrecho margen de luminosidad. Este es el caso de Hildenbrandia rivularis, que presenta un muy bajo punto de saturación $\left(100 \mu \mathrm{E} \mathrm{m} \mathrm{s} \mathrm{s}^{-1}\right.$ según Kremer, 1983). Audouinella violacea se deteriora rápidamente bajo elevadas intensidades de luz (RIDER \& W AGNER, 1972).

\section{AGRADECIMIENTOS}

Agradecemos a Isabel Muñoz (Dept. Ecologia, Univ. Barcelona) la recolección de Thorea ramosissima, así como los datos fisico-químicos de las aguas en que fue hallada. El Dr. Xavier Llimona ha hecho interesantes sugerencias en un primer manuscrito.

\section{Bibliografía}

AвоAL, M., 1987. Flora algal epicontinental de la cuenca del rio Segura, SE de España. Tesis doctoral, Facultad de Biología, Universidad de Murcia, 350 pp.

apha (American Public Health association), 1980. Standard methods for examination of water and wastewater. 15 th edition. Washington, $1134 \mathrm{pp}$.

Battiatio, A., M. Cormaci, G. Furnari \& E. Lanfranco, 1979. Compsopogon coeruleus (Balbis) Montagne (Rhodophyta, Bangiophycideae) in Malta and of Compsopogon chalybeus Kutzing in an aquarium at Catania (Sicily). Rev. Algol., N.S. 14: 11-16.

BourRelly, P., 1955. Quelques stations françaises d'Hildenbrandia rivularis (Liebm.) Breb. Rev. Algol. 1: 168-169.

Bourrelly, P., 1970. Les algues d'eau douce. Initiation a la systématique. III. Les algues bleues et rouges. Les eugléniens, peridiniens et cryptomonadines. Boubée, París. $511 \mathrm{pp}$.

Busouets, J.M., C. Picado \& M.C. Hernández Mariné, 1985. Compsopogon coeruleus (Balbis) Montagne (Rhodophyta, Bangiophyceae). Ampliación de su área de distribución en la Península Ibérica. Collect. Bot. 16(1):229-230. 
CABAllero, S., 1929. Datos sobre la flora algológica de Guadalajara (3" parte). Bol. R. Soc. Esp. Hist. Nat. 29: 315-324.

CAmbra, J., 1985. Catàlegs de les especies de cianofícies, d'algues continentals i del plàncton marí dels Països Catalans. Annex I: 501-522. In: Historia Natural dels Països Catalans. Plantes inferiors. Vol. 4, Llimona, X, et al., Ed. Enciclopedia Catalana, Barcelona. $588 \mathrm{pp}$

CAmbra, J., 1986. Aproximació a la flora $i$ a la vegetació de les algues d'aigües epicontinentals de la Península del Cap de Creus (Alt Empordà). Tesis de licenciatura. Facultat de Biologia, Universitat de Barcelona.

CAmbra, J., 1987. Lemanea nodosa Kütz. 1843 en el torrente de Arbúcies (Barcelona). Act. VI Simp. Nac. Bot. Criptog.: 19-24.

Descy, J.P., 1973. Étude quantitative du peuplement algal benthique en vue de l'établissement d'une methodologie d'estimation biologique de la qualite des eaux courantes. Application au cours belge de la Meuse et de la Sambre. In: Recherche et technique au service de l'environment. $\mathrm{CE}$ BEDOC: 159-206, Liège.

Friedrich, G. \& D. Muller, 1984. Rhine. In: B.A. Whitton (Ed.), Ecology of European rivers. Blackwell Sc. Publ. Oxford: 265-315.

FrITSCH, F.E., 1929. The encrusting algal communities of certain fast-flowing streams. New Phytologist 28(3): 165-197.

GONZÁleZ FrAgOSO, R., 1883. Apuntes para la flora de Sevilla (Criptogamia). An. Soc. Esp. Hist. Nat. 12: 393-420.

GonzÁlez GuERrero, P., 1946. Algas invernales gaditanas. An. Jard. Bot. Madrid 7: 433-456.

Hamel, G., 1924, 1925. Floridées de France, Revue Algologique 1, 2: 278-292, 427-457; 39-67, 280-309.

KANN, E., 1978. Systematik und okologie der Algen Österreischischer Bergbache. Arch. Hydrobiol. Suppl. 53: 405-643

KREMER, B.P., 1983. Untursuchungen zur Ökophysiologie eininger Süswasserrotalgen. Decheniana (Bonn) 136: 31-42.

LacoizqueTA, J.M., 1885. Plantas espontáneas del valle de Vertizarana (Navarra), II. An. Soc. Esp. Hist. Nat. 14: 185-238.

Lewin, M., 1888. Ueber Spanische Süswasseralgen. Bih. Svensk. Vedensk. Akad. Handl. Avd. 14(III): 1-24

MARGAlEF, R., 1950. Datos para la hidrobiología de la Cordillera Cantábrica, especialmente de los Picos de Europa Publ. Inst. Biol. Apl. 7: 37-76.
Margalef, R., 1951. Materiales para la hidrobiología de la Isla de Ibiza. Publ. Inst. Biol. Apl. 8: 5-70.

MARGAlef, R., 1955. Materiales para una flora de las algas del NE de España. VI. Desmidiales, Rhodophyceae. Collect. Bot. 4(3): 319-330.

REIS, P.M., 1974. Chaves para a identificaçao das espécies portuguesas de Batrachospermum Roth. An. Soc. Broteriana 40: $37-125$.

RIDER, D.E. \& R.H. WAGNER, 1972. The relationship of light, temperature and current to the seasonal distribution of Batrachospermum (Rhodophyta). J. Phycol. 8: 323-331.

SABATER, S., 1987. Estudi de les poblacions d'algues del riu Ter. Tesis doctoral. Facultat de Biologia, Universitat de Barcelona.

SCANNELL, M.P.J., 1972. Algal paper of Oedogonium sp., its occurrence in the Burren, Co. Clare. Ir. Nat. J. 17(5): 147-152.

ShEATH, R.G., 1984. The biology of freshwater red algae. In: Round, F.E. \& Chapman, D.J. (Eds.), Progress in phycological research 3: 89-157. Biopress Ltd.

SheATH, R.G. \& K.M. Cole, 1984. Systematics of Bangia (Rhodophyta) in North America. I. Biogeographic trends in morphology. Phycologia 23: 383-396.

Skuja, H., 1938. Comments on fresh-water Rhodophyceae. Bot. Rev. 4: 665-676.

StARMACH, K., 1963. Hildenbrandia rivularis and Chamaesiphon fuscoviolaceus and accompanying algae in the stream Lubogozcz in the Beskid Wyspowy. Fragm. Florist. Geobot. Ann. 15(4): 487-501.

Starmach, K., 1969. Hildenbrandia rivularis and associating algae in the stream Cedronka near Wejherowa. Fragm. Florist. Geobot. Ann. 15(3): 143-150.

STARMACH, K., 1977. Phaeophyta-Brunatnice and Rhodophyta-Krasnorosty, Flora Slodkowodna Polski 14. Ed. Polska Academia, Warszawa. 425 pp.

Tomas, X., 1981. Thorea ramosissima en un canal del litoral valenciano. Fol. Bot. Misc. 2: 71-74

Tomas, X., P. Lopez, R. M argalef-Mir \& F.A. Comin, 1980. Distribution and ecology of Compsopogon coeruleus (Balbis) Montagne (Rhodophyta, Bangiophyceae) in Eastern Spain. Cryptogamie, Algologie 1(3): 179-186.

WeHr, J.D. \& J.R. STEIN, 1985. Studies on the biogeography and ecology of the freshwater Phaeophycean alga Heribaudiella fluviatilis. J. Phycol. 21: 81-93.

Whitton, B., 1975. Algae. In: Whitton, B.A. (Ed.), River Ecology, Univ. California Press: 81-105. 